\title{
Angelicin inhibits human lung carcinoma A549 cell growth and migration through regulating JNK and ERK pathways
}

\author{
GUANGCAI LI ${ }^{1}$, YUAN HE ${ }^{2}$, JUN YAO ${ }^{2}$, CHUYING HUANG $^{1}$, XIUSHENG SONG ${ }^{2}$,

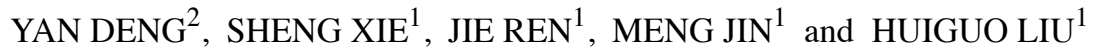 \\ ${ }^{1}$ Department of Respiratory Diseases, Tongji Hospital, Key Laboratory of Pulmonary Diseases of Health Ministry, \\ Tongji Medical College, Huazhong University of Science and Technology, Wuhan, Hubei 430000; \\ ${ }^{2}$ The Central Hospital of Enshi Tujia and Miao Autonomous Prefecture, Enshi, Hubei 445000, P.R. China
}

Received April 7, 2016; Accepted August 16, 2016

DOI: $10.3892 /$ or.2016.5166

\begin{abstract}
Angelicin is a member of a well-known class of chemical photosensitizes that have anticancer properties in several cancer cell lines. However, the effects and the potential underlying mechanisms of angelicin action on human lung cancer cells remain unclear. Here, we report that angelicin has an essential role in inhibiting human lung carcinoma growth and metastasis. We found that angelicin markedly induced cell apoptosis and arrested the cell cycle in vitro. Angelicin also inhibited the migration of non-small cell lung cancer (NSCLC) A549 cells in a Transwell assay in a dose-dependent manner. In addition, after angelicin treatment, the expression levels of Bax, cleaved caspase- 3 and cleaved caspase- 9 were increased, and $\mathrm{Bcl}-2$ expression was decreased. Moreover, our results indicate that angelicin inhibits NSCLC growth not only by downregulating cyclin B1, cyclin E1 and $\mathrm{Cdc} 2$, which are related to the cell cycle, but also by reducing MMP2 and MMP9 and increasing E-cadherin expression levels. Furthermore, extracellular signal-regulated kinase (ERK)1/2 and c-Jun $\mathrm{NH}_{2}$-terminal protein kinase $(\mathrm{JNK}) 1 / 2$ phosphorylation increased in parallel with the angelicin treatments. The inhibition of ERK1/2 and JNK1/2 by specific inhibitors significantly abrogated angelicin-induced cell apoptosis, cell cycle arrest and migration inhibition. We established in vivo A549 cell transplant and metastasis models and found that angelicin exerted a significant inhibitory effect on A549 cell growth and lung metastasis. Overall, our results suggested that angelicin is able to inhibit NSCLC A549 cell growth and metastasis by targeting ERK and JNK signaling, which demonstrates potential for NSCLC therapy.
\end{abstract}

Correspondence to: Professor Huiguo Liu, Department of Respiratory Diseases, Tongji Hospital, Key Laboratory of Pulmonary Diseases of Health Ministry, Tongji Medical College, Huazhong University of Science and Technology, Wuhan, Hubei 430000, P.R. China

E-mail: hgliu@tjh.tjmu.edu.cn

Key words: angelicin, A549 cells, proliferation, apoptosis, metastasis

\section{Introduction}

Lung cancer is one of the most common malignant tumors and is a major cause of global morbidity and mortality. Even with recent advances in diagnosis and clinical treatment, the five-year survival rate is only $15 \%$ (1). Non-small cell lung cancer (NSCLC), such as the A549 cell line, accounts for $85 \%$ of all lung cancer cases (2). Notably, most patients with NSCLC are diagnosed after having reached a terminal stage and developed metastases in adjacent or distant organs (3). Systemic pharmacotherapy is the primary treatment for these patients (4). Recently, drugs targeting key pathways involved in NSCLC have generated new approaches for treating this condition. However, efficacious, curative drug therapies for NSCLC and its metastases remain elusive (5). There is an urgent need for more effective agents for the clinical treatment of NSCLC.

Tumor development involves abnormal cell proliferation and migration (6). Additionally, cell proliferation is closely related to the cell cycle; therefore, inducing apoptosis, arresting the cell cycle and inhibiting metastasis are effective methods of controlling tumor cell growth (7). Many cytokines and signaling pathways, including Bax, Bcl-2, matrix metalloproteinases (MMPs), cyclins, AKT, mitogen-activated protein kinase (MAPK), c-Jun $\mathrm{NH}_{2}$-terminal protein kinase (JNK) and extracellular signal-regulated kinase (ERK), play influential roles in regulating the abnormal proliferation and migration of tumor cells (8-10). These molecular players and signaling cascades are involved in regulating apoptosis, migration and the cell cycle.

Angelicin is a traditional Chinese medicine and a well-known furocoumarin that has been a common treatment for a long time (11). Recently, it has been used to treat various skin diseases $(12,13)$. Moreover, previous studies have demonstrated that angelicin has potential for curing leukemia by inhibiting tumor cell proliferation (14). Furthermore, angelicin is reportedly a potential candidate for treating neuroblastoma by inducing cell apoptosis (11). However, there have been few studies on the effect of angelicin on NSCLC (15).

In this study, we aimed to assess the abrogation of A549 cell growth resulting from angelicin inducing apoptosis, arresting the cell cycle and inhibiting metastasis. To gain insight into the potential anticancer mechanism of angelicin, we further 
investigated its effects on growth and related metastasis signaling pathways. Our results suggest that angelicin inhibits A549 cell activity by regulating ERK and JNK signaling as well as related metastasis signaling. All the results suggest that angelicin could be an effective therapeutic candidate for NSCLC intervention.

\section{Materials and methods}

Ethics statement. All experiments were approved by the Huazhong University of Science and Technology Committee and the Tongji Medical College Ethics Committee, Tongji Hospital (Wuhan, China).

Reagents. Dimethyl sulfoxide (DMSO), angelicin and 3-(4,5-dimethylthiazol-2-yl)-2,5-diphenyltetrazolium bromide (MTT) were purchased from Sigma-Aldrich, Invitrogen Life Technologies (Carlsbad, CA, USA). Polyclonal antibodies against Bax, Bcl-2, caspase-3, caspase-9, cyclin B1, cyclin E1, Cdc2, E-cadherin, MMP2, MMP9, p-ERK1/2, ERK1/2, p-JNK1, JNK1, P-p38 MAPK, p38 MAPK, Akt and p-Akt were purchased from Cell Signaling Technology, Inc. (Danvers, MA USA). GAPDH, goat anti-mouse IgG and goat anti-rabbit IgG were purchased from Proteintech Group, Inc. (Rosemont, IL, USA). Dulbecco's modified Eagle's medium (DMEM), fetal bovine serum (FBS), penicillin and streptomycin were purchased from Gibco, Invitrogen Life Technologies (Carlsbad, CA, USA). A protease inhibitor cocktail was purchased from Roche Diagnostics (Basel, Switzerland). A BCA protein assay reagent kit and an enhanced chemiluminescence (ECL) plus reagent kit were obtained from Pierce Biotechnology, Inc. (Rockford, IL, USA).

Cell lines and culture. Human lung cancer A549 cells were purchased from the Chinese Academy of Sciences Cell Bank (CBP60084; Shanghai, China) and were maintained in RPMI-1640 with 10\% FBS and antibiotics (penicillin and streptomycin) in an incubator with a humidified atmosphere $\left(5 \% \mathrm{CO}_{2}, 37^{\circ} \mathrm{C}\right)$.

MTT assay. An MTT colorimetric assay was performed according to the manufacturer's instructions. In brief, exponentially growing cells were seeded in 96-well plates at a density of $5 \times 10^{3}$ cells/well. Following an overnight incubation, the cells were treated with various doses of angelicin for $24 \mathrm{~h}$ at $37^{\circ} \mathrm{C}$. Then, the medium was discarded, and MTT $(0.5 \mathrm{mg} / \mathrm{ml})$ was added into each well and incubated for $4 \mathrm{~h}$ at $37^{\circ} \mathrm{C}$. Subsequently, the MTT-containing medium was removed and replaced with $150 \mu \mathrm{l}$ of DMSO. The absorbance at $570 \mathrm{~nm}$ was then determined using a Bio-Rad Model 680 microplate reader (Bio-Rad Laboratories, Inc., Hercules, CA, USA). The $\mathrm{IC}_{50}$ was calculated from the MTT dose-response curves of cell viability against drug concentration. Three replicate wells were used for each analysis.

TdT-mediated dUTP nick end labeling (TUNEL) assay. A TUNEL assay was utilized to analyze the pro-apoptotic effect of angelicin in A549 cells. After being appropriately treated with angelicin, cells were fixed in $4 \%$ paraformaldehyde for 30 min and then permeabilized with $0.05 \%$ Triton X-100 on ice for $5 \mathrm{~min}$. The cells were subjected to TUNEL while being incubated in a humidified chamber at $37^{\circ} \mathrm{C}$ for $60 \mathrm{~min}$ in the dark. The cells were washed 3 times with phosphate-buffered saline (PBS; pH 7.4); anti-fluorescence quenching solution was then added and allowed to react for $5 \mathrm{~min}$. Finally, the cells were examined using a confocal laser scanning microscope.

Flow cytometric apoptosis assay. To detect the apoptotic effects of angelicin on A549 cells, an Annexin V-FITC/propidium iodide (PI) apoptosis detection kit was used. In brief, A549 cells were seeded in a 6-well plate and incubated for $24 \mathrm{~h}$; the cells were then treated with the DMSO control or angelicin $(10,25$ or $50 \mu \mathrm{mol})$ for $24 \mathrm{~h}$. Next, the cells were collected, washed with PBS, and resuspended in $100 \mu \mathrm{l}$ of $1 \mathrm{X}$ binding buffer. Annexin V-FITC/PI were added to each group, and the cells were incubated for $15 \mathrm{~min}$ at room temperature in the dark. The cells were then analyzed by flow cytometry (BD Calibur; BD Biosciences, San Jose, CA, USA). Each experiment was performed 3 times.

Flow cytometric cell cycle analysis. Flow cytometry used to examine the effect of angelicin on the cell cycle. After being treated with the DMSO control or angelicin $(10,25$ or $50 \mu \mathrm{mol})$ for $24 \mathrm{~h}$, A549 cells were harvested, washed twice with PBS (pH 7.4) and fixed with $70 \%$ ethanol for $20 \mathrm{~min}$. Then, the cells were centrifuged $(300 \mathrm{x} \mathrm{g}, 5 \mathrm{~min})$ to eliminate the ethanol, washed twice with PBS (pH 7.4) and stained with PI in the dark for $30 \mathrm{~min}$. Finally, the cell cycle distribution was assessed by flow cytometry (BD Calibur; BD Biosciences). Each experiment was performed 3 times.

Wound-healing assay. The anti-migratory effects of angelicin on A549 cells were examined through a wound-healing assay. After attached cells had grown to $90 \%$ confluence, a wound in the monolayer was created using a pipette tip, and the cells were washed twice with PBS ( $\mathrm{pH} 7.4)$. Then, the cells were treated with the DMSO control or angelicin $(10,25$ or $50 \mu \mathrm{mol})$ for $24 \mathrm{~h}$. The number of migrated cells was determined using an inverted microscope. Five randomly chosen fields were analyzed in each well.

Transwell migration assay. Transwell chambers (Corning Costar, Cambridge, MA, USA) were used for the cell migration assays. A549 cells $\left(1 \times 10^{5}\right)$ were seeded in the top chamber with FBS-free medium. Culture medium containing the DMSO control or angelicin $(10,25$ or $50 \mu \mathrm{mol})$ was added to the bottom chamber. After the cells were incubated for $24 \mathrm{~h}$ at $37^{\circ} \mathrm{C}$, the upper side of the membrane was removed, and the cells in the lower chamber were fixed in $4 \%$ paraformaldehyde for $15 \mathrm{~min}$. The fixed cells were washed with PBS ( $\mathrm{pH} 7.4) 3$ times and then stained with $0.25 \%$ crystal violet for $5 \mathrm{~min}$. Cell migration was evaluated using an inverted microscope (x200). Six randomly chosen fields were analyzed in each group and presented as the mean of 3 independent experiments.

Western blotting. After the cells were treated with the DMSO control or angelicin, proteins from the A549 cell lysates were extracted and $12 \%$ SDS-PAGE was used to separate the protein samples. Then, the proteins were transferred to a PVDF membrane, which was blocked with $5 \%$ skim milk and incubated 
A

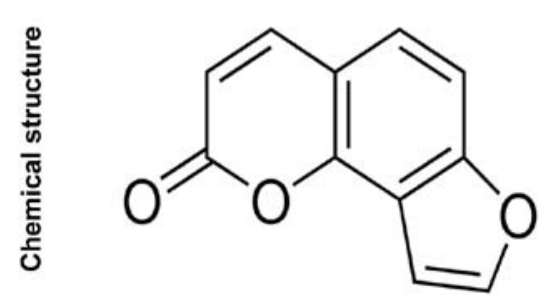

Angelicin
B

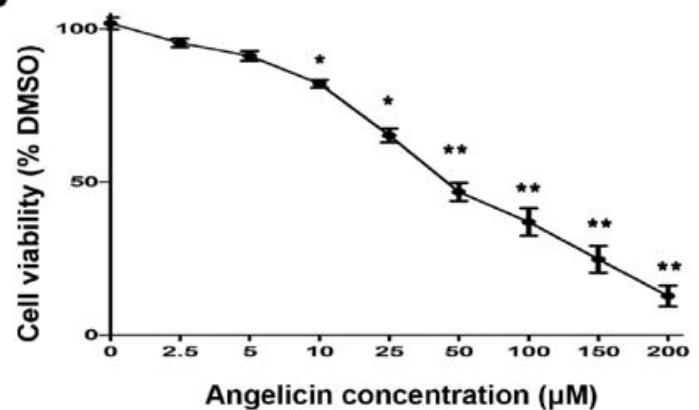

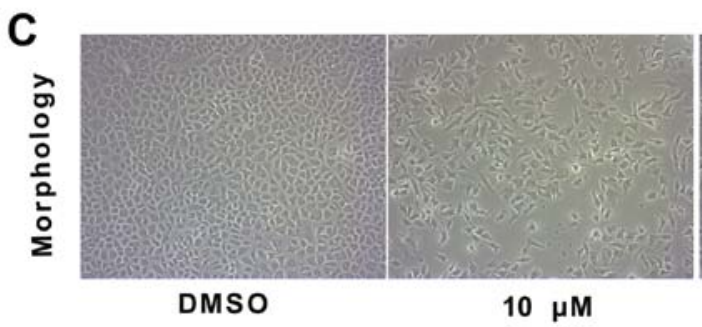

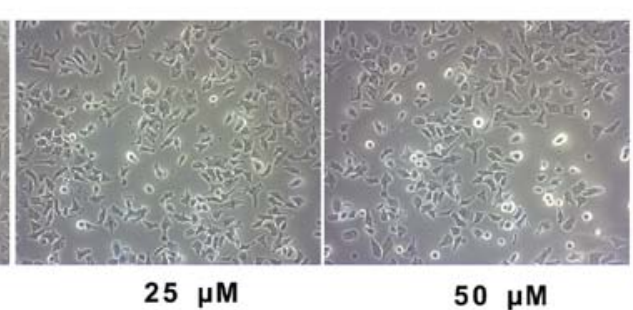

Figure 1. Cytotoxic effect of angelicin on A549 cells. (A) Chemical structure of angelicin. (B) MTT assay was performed to select the appropriate doses of angelicin for subsequent experiments. (C) Inverted microscope observation of A549 cell morphology (x200 magnification). Data are presented as the mean \pm standard deviation obtained from 3 independent experiments. ${ }^{*} \mathrm{P}<0.05,{ }^{* *} \mathrm{P}<0.01$ compared with $\mathrm{DMSO}, \mathrm{n}=4$.

with different antibodies overnight at $4^{\circ} \mathrm{C}$. The antibodies were diluted to the following concentrations: $\operatorname{Bax}(1: 1,000)$, Bcl-2 (1:2,000), caspase-3 (1:1,000), caspase-9 (1:800), cyclin B1 $(1: 1,000)$, cyclin E1 $(1: 1,000)$, Cdc2 $(1: 2,000)$, E-cadherin (1:2,000), MMP2 (1:800), MMP9 (1:800), p-ERK1/2 (1:2,000), ERK1/2 (1:2,000), p-JNK1 $(1: 1,000)$, JNK1 (1:1,000), P-p38 MAPK (1:1,000), p38 MAPK (1:1,000), Akt $(1: 2,000)$ and p-Akt (1:1,000). After being washed 3 times in TBST, the membrane was incubated with HRP-conjugated secondary antibodies for $1 \mathrm{~h}$ at room temperature. An ECL kit was used to develop the immuno-reactive bands.

Animal groups and in vivo xenograft study. For the A549 xenograft studies, female nude mice aged 5 weeks were used. A549 cells $\left(5 \times 10^{6}\right)$ were subcutaneously implanted in the right flank of the mice. The tumor-bearing mice were randomly divided into two groups: the PBS ( $\mathrm{pH}$ 7.4) control group and the angelicin group. Mice in the angelicin group were treated with angelicin for 4 consecutive weeks by oral gavage $(100 \mathrm{mg} / \mathrm{kg})$, while mice in the control group received PBS (pH 7.4). Tumor volume and body weight were monitored once every 2 days. At the end of 4 weeks, the mice were sacrificed and the tumor xenografts were removed and measured.

Experimental lung metastasis experiments. Male nude mice (SIPPR-BK Laboratory Animal Co., Ltd., Shanghai, China) aged 5 weeks were used. A549 cells $\left(5 \times 10^{6}\right)$ were injected via the tail vein. Two weeks later, the mice were randomly divided into two groups: the PBS ( $\mathrm{pH}$ 7.4) control group and the angelicin (100 mg/kg/day) group. Mice in the angelicin group received an intragastric administration of angelicin dissolved in PBS ( $\mathrm{pH} 7.4$ ) every day for 4 weeks. The animals were sacrificed after this period. The lungs were then removed and fixed in Bouin's solution for $4 \mathrm{~h}$, and the number of metastatic lesions was determined macroscopically.
Immunohistochemistry. Sections of the lung specimens were deparaffinized and rehydrated. Then, the sections were rinsed in PBS (pH 7.4) 3 times and incubated in 3\% hydrogen peroxide for $15 \mathrm{~min}$ at room temperature. After being blocked in $10 \%$ goat serum for $30 \mathrm{~min}$, the tissue sections were incubated overnight at $4^{\circ} \mathrm{C}$ with polyclonal antibodies (MMP2, 1:100; MMP9, 1:80; and E-cadherin, 1:50). The sections were subsequently incubated with peroxidase-conjugated goat anti-rabbit secondary antibody (1:100) for $1 \mathrm{~h}$ at room temperature, washed 5 times with PBS ( $\mathrm{pH} 7.4)$, and visualized with the peroxidase substrate diaminobenzidine (DAB) under a microscope.

Statistical analysis. The data shown in this study were obtained from at least 3 independent experiments and analyzed using SPSS 16.0 (SPSS, Inc., Chicago, IL, USA). Statistical significance was determined using an unpaired Student's t-test. All results are presented as the mean \pm standard deviation (SD). $\mathrm{P}<0.05$ was considered statistically significant vs. the control group.

\section{Results}

Angelicin inhibits the growth of A549 cells. The chemical structure of angelicin is shown in Fig. 1A. To examine the cytotoxic effects of angelicin on A549 cells, an MTT assay was performed. A549 cells were treated with different concentrations of angelicin $(0,2.5,5,10,25,50,100,150$ and $200 \mu \mathrm{mol} / \mathrm{l})$ for $24 \mathrm{~h}$. As shown in Fig. 1B, the proliferation of angelicin-treated A549 cells was markedly suppressed at $24 \mathrm{~h}$ compared with that of cells in the control group. These results showed that the angelicin treatment suppressed A549 cell proliferation in a dose-dependent manner. As the $\mathrm{IC}_{50}$ value for angelicin was $50.14 \mu \mathrm{mol}$, doses of 10,25 and $50 \mu \mathrm{mol}$ angelicin were selected for use in subsequent experiments to assess A549 cell growth suppression. 

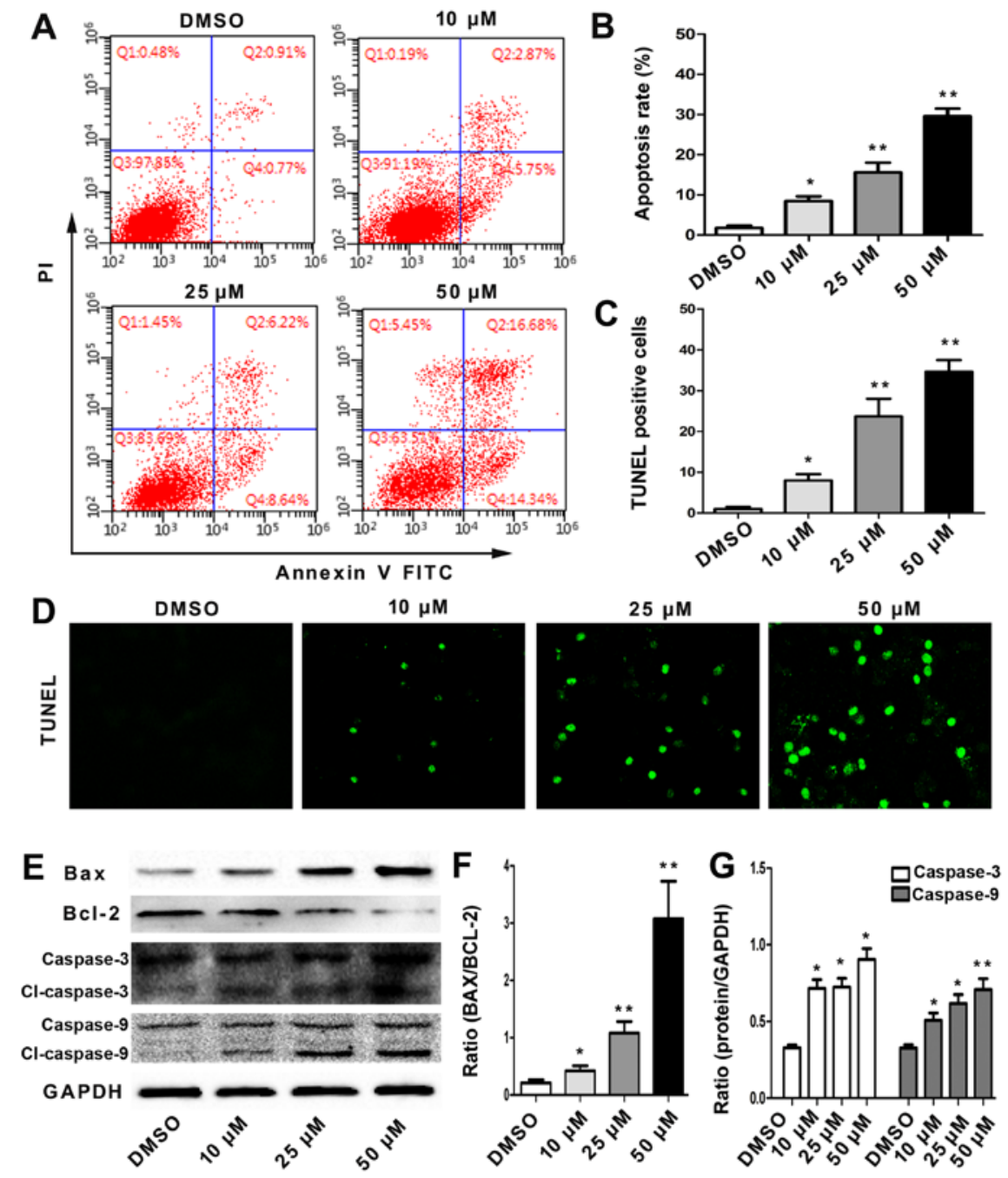

Figure 2. Angelicin induces apoptosis in A549 cells. (A) Representative flow cytometry scatter plots of PI (y-axis) vs. Annexin V-FITC (x-axis) and (B) quantitation data of the average of 3 independent flow cytometry experiments. (C) Bar chart showing quantitation data of the average of 3 independent TUNEL assay experiments. (D) TUNEL assay was performed to measure the ratio of apoptotic cells. TUNEL-positive cells were counted from at least 100 random fields. (E) The expressions of Bax, Bcl-2, caspase-3 and caspase-9 were analyzed with western blotting and ( $\mathrm{F}$ and $\mathrm{G})$ the proteins expression were evaluated. ${ }^{*} \mathrm{P}<0.05,{ }^{* *} \mathrm{P}<0.01$ compared with DMSO, $\mathrm{n}=3$. PI, propidium iodide; FITC, fluorescein isothiocyanate.

Additionally, the morphological changes of angelicin-treated cells were assessed using an inverted microscope (Fig. 1C). The results showed that the angelicin treatment caused marked morphological alterations, including the adherence of cells in poor condition, reduced cell volume, chromatin condensation, karyopyknosis and nuclear fragmentation. Furthermore, as the dose of angelicin increased, the morphological alterations became more apparent.

Angelicin induces apoptosis and regulates the expression of apoptosis-associated proteins in A549 cells. To assess whether angelicin-induced cell growth suppression was associated with cell apoptosis, the effects of angelicin on apoptosis were evaluated by flow cytometry using Annexin V-FITC/PI double staining. As shown in Fig. 2A and B, the percentage of early- and late-stage apoptotic cells increased in a dose-dependent manner. This result showed that the angelicin treatment caused a significant increase in apoptosis.

Moreover, the extent of A549 cell apoptosis was examined using TUNEL staining. The TUNEL assay revealed only $2 \pm 2 \%$ TUNEL-positive nuclei in the DMSO control cells, and in accordance with the flow cytometric analysis, a significantly increased percentage of TUNEL-positive nuclei was observed in the cells incubated with angelicin (Fig. 2C and D). In addition, angelicin-induced apoptosis occurred in a dose-dependent manner.

Furthermore, to gain insight into the potential pro-apoptotic mechanisms of angelicin, the protein expression levels of Bax, Bcl-2, caspase-3 and caspase-9, which are important apoptosis-associated proteins, were detected by western blotting. As shown in Fig. 2E-G, the angelicin treatment markedly induced Bax expression to a level comparable to that of the control cells and significantly reduced Bcl-2 expression in a dose-dependent manner. In addition, the angelicin treatment promoted the expression of cleaved caspase- 3 and caspase- 9 in a dose-dependent manner, demonstrating the pro-apoptotic effect of angelicin. All the results demonstrated that the pro-apoptotic effect of angelicin may be associated with the regulation of these proteins.

Angelicin arrested the cell cycle and regulated related proteins. The effect of angelicin on the cell cycle distribution was 

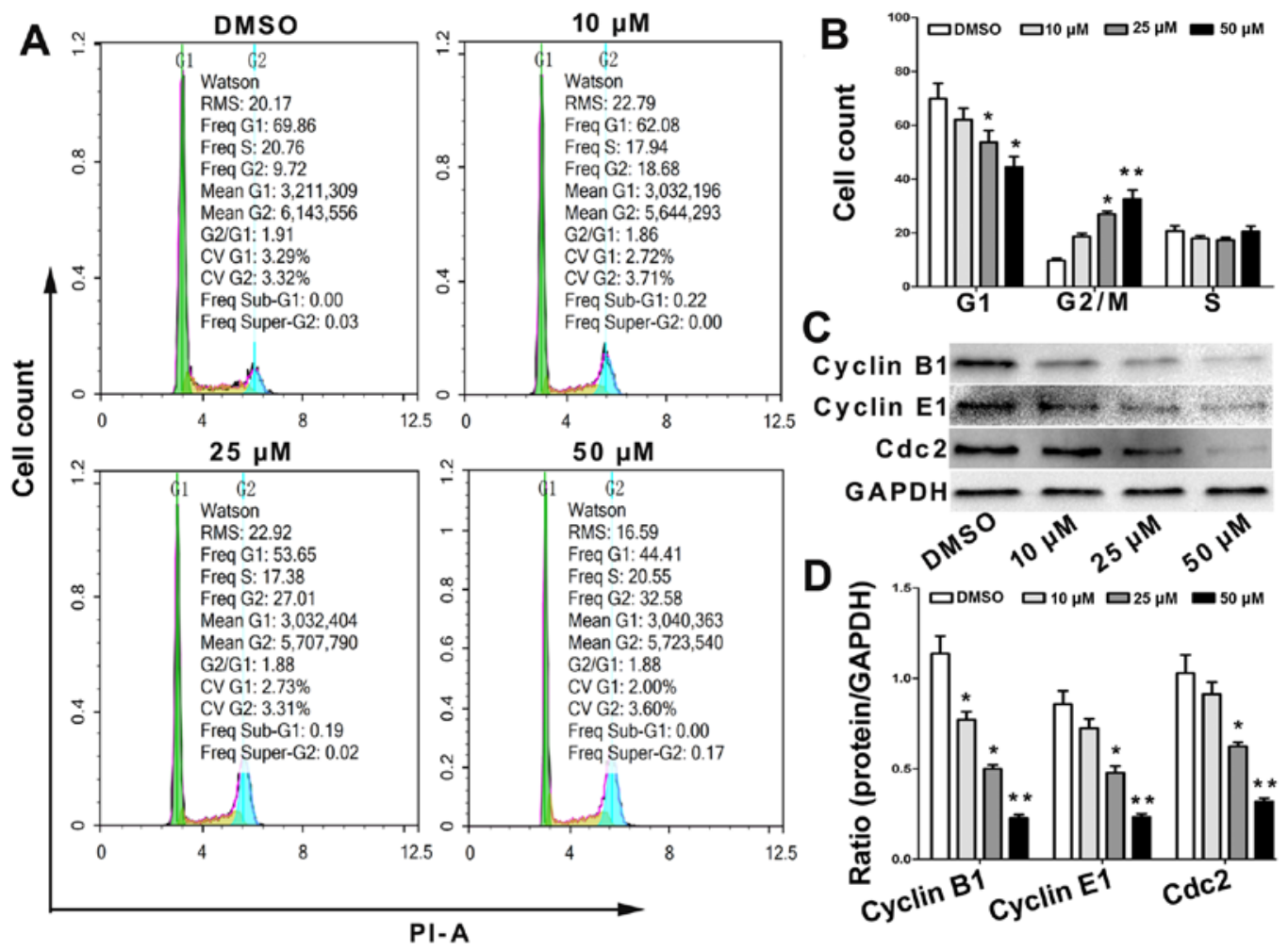

Figure 3. Angelicin arrested the A549 cell cycle at the G2/M phase. A549 cells were treated with increasing concentrations of angelicin for $24 \mathrm{~h}$. (A) Representative flow cytometry cell cycle of PI. (B) Quantitation data of flow cytometry cell cycle of 3 independent experiments and (C) effect of angelicin on cycle proteins expression of cyclin B1, cyclin E1 and Cdc2 by western blotting. (D) Quantitation data of (C). "P<0.05, ${ }^{* *} \mathrm{P}<0.01$ compared with DMSO, n=3 PI, propidium iodide.

determined using flow cytometry. Cells were or were not treated with angelicin at 10,25 and $50 \mu \mathrm{mol}$ for $24 \mathrm{~h}$ and were then stained with PI. The results showed that the proportion of cells in the $\mathrm{G} 2 / \mathrm{M}$ and $\mathrm{G} 0 / \mathrm{G} 1$ phase increased and decreased, respectively, in a dose-dependent manner (Fig. 3A and B).

To further elucidate the specific regulatory proteins responsible for the cell cycle arrest, we explored the effect of angelicin on the regulatory proteins cyclin B1, cyclin E1 and Cdc2. As shown in Fig. 3C and D, the levels of cyclin B1, cyclin E1 and Cdc2 were significantly downregulated in the angelicin-treated cells. These data revealed that alterations in the expression levels of cell cycle regulatory proteins and the arrest of cell growth in the $\mathrm{G} 2 / \mathrm{M}$ phase are involved in angelicin-induced changes in cell cycle progression.

Angelicin inhibits A549 cell migration and invasion. Tumor cell migration and invasion are key steps in cancer metastasis (16). To evaluate the potential effect of angelicin on A549 cell migration, we assessed alterations in cell mobility using a scratch test and a Transwell assay. The results showed that the angelicin treatment significantly decreased A549 cell migration capability in a dose-dependent manner (Fig. 4A and B). The Transwell assay results showed that the migration and invasion of the angelicin-treated A549 cells were significantly inhibited in a dose-dependent manner compared with that of the control cells (Fig. 4C and D).

E-cadherin, MMP2 and MMP9 are responsible for cell migration, invasion and cell-matrix adhesion, particularly in the process of cancer metastasis (17). Therefore, we detected the effects of angelicin on the expression of these proteins using western blotting. As shown in Fig. 4E and F, we observed that angelicin strongly increased E-cadherin expression but reduced MMP2 and MMP9 expression in A549 cells in a dose-dependent manner compared with that of the control cells. These results indicate that angelicin directly inhibits the migration and invasion of A549 cells.

Angelicin inhibits A549 cell growth and migration via ERK and JNK pathways. The MAPK and Akt signaling pathways have important roles in regulating tumor cell apoptosis, cell cycle progression and metastasis (17). To further explore the mechanism underlying angelicin-induced apoptosis, cell cycle arrest and migration inhibition in A549 cells, we evaluated whether angelicin modulates the MAPK and Akt signaling pathways when affecting A549 cells. We first examined the activation status of p38 MAPK, JNK, ERK and AKT by western blotting with antibodies specific to the phosphorylated forms of these kinases. As shown in Fig. 5A-C, treating A549 cells with angelicin significantly increased the levels of phosphorylated JNK and ERK compared with the total protein expression levels in a dose-dependent manner. However, angelicin had no effect on the phosphorylation of p38 MAPK, AKT, or the total expression levels of these proteins. These results suggest that angelicin may activate the JNK and ERK pathways in A549 cells.

We next tested whether SP600125, a JNK inhibitor, or U0126, an ERK inhibitor, could reverse the angelicin-induced apoptosis, cell cycle arrest and migration inhibition in A549 cells. First, the levels of phosphorylated JNK and ERK were analyzed by western blotting. The SP600125 and U0126 

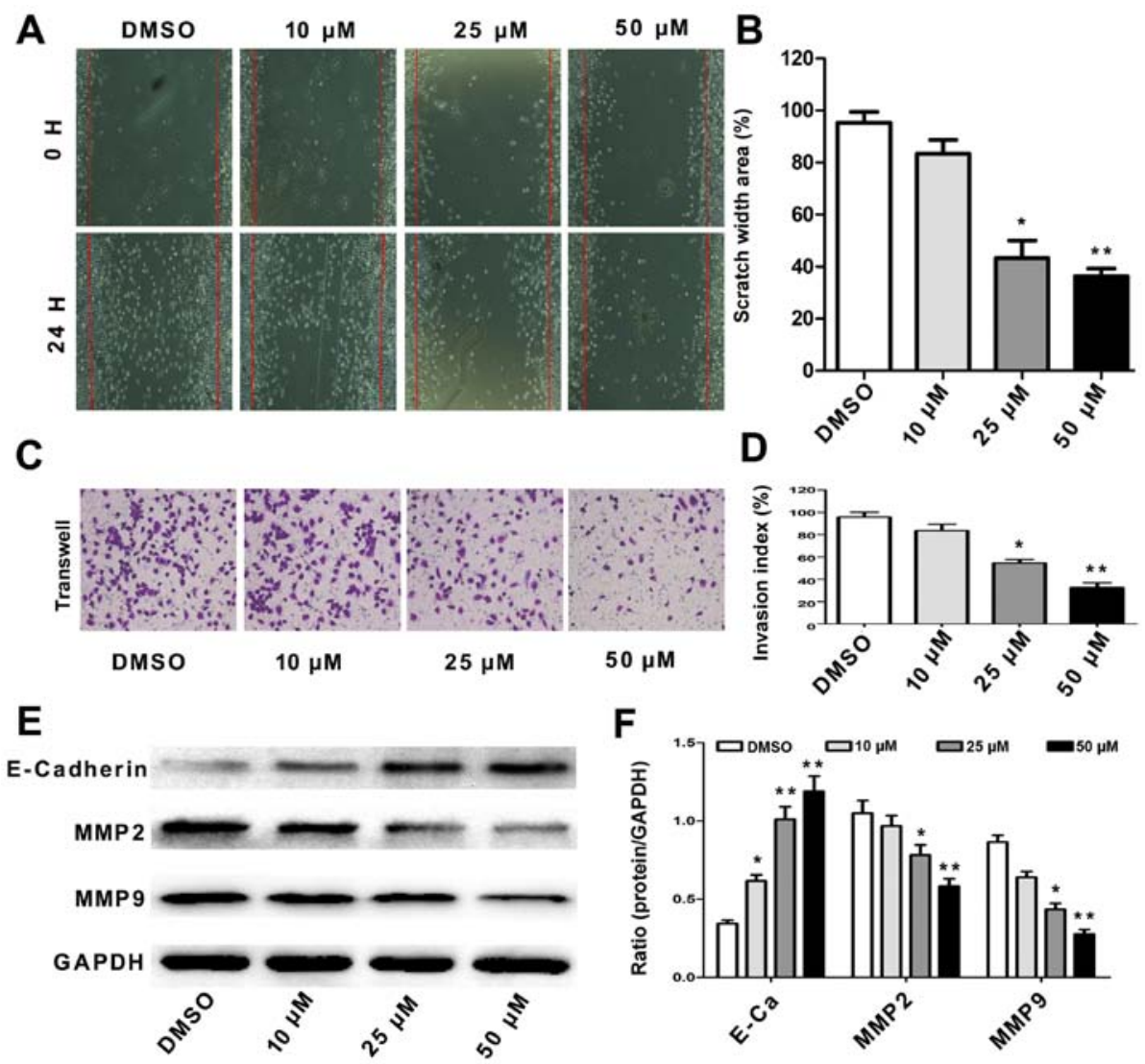

Figure 4. Angelicin inhibits migration, adhesion and invasion of A549 cells. (A) A monolayer of cells was scraped with a pipette tip and then treated with angelicin. The migrating cells were assessed with a microscope equipped with a camera (x200 magnification), and (B) quantitation data of (A). (C) The invasive ability was evaluated by a Transwell invasion assay (x200 magnification), and (D) migratory cells in the bottom surface of the membrane were counted. (E) Full-length blots of E-cadherin, MMP2, MMP9 and GAPDH are presented. (F) Results were quantified by densitometry analysis of the bands from (E) and then normalization to GAPDH protein. ${ }^{*} \mathrm{P}<0.05,{ }^{* *} \mathrm{P}<0.01$ compared with DMSO, $\mathrm{n}=3$. MMP, matrix metalloproteinase.
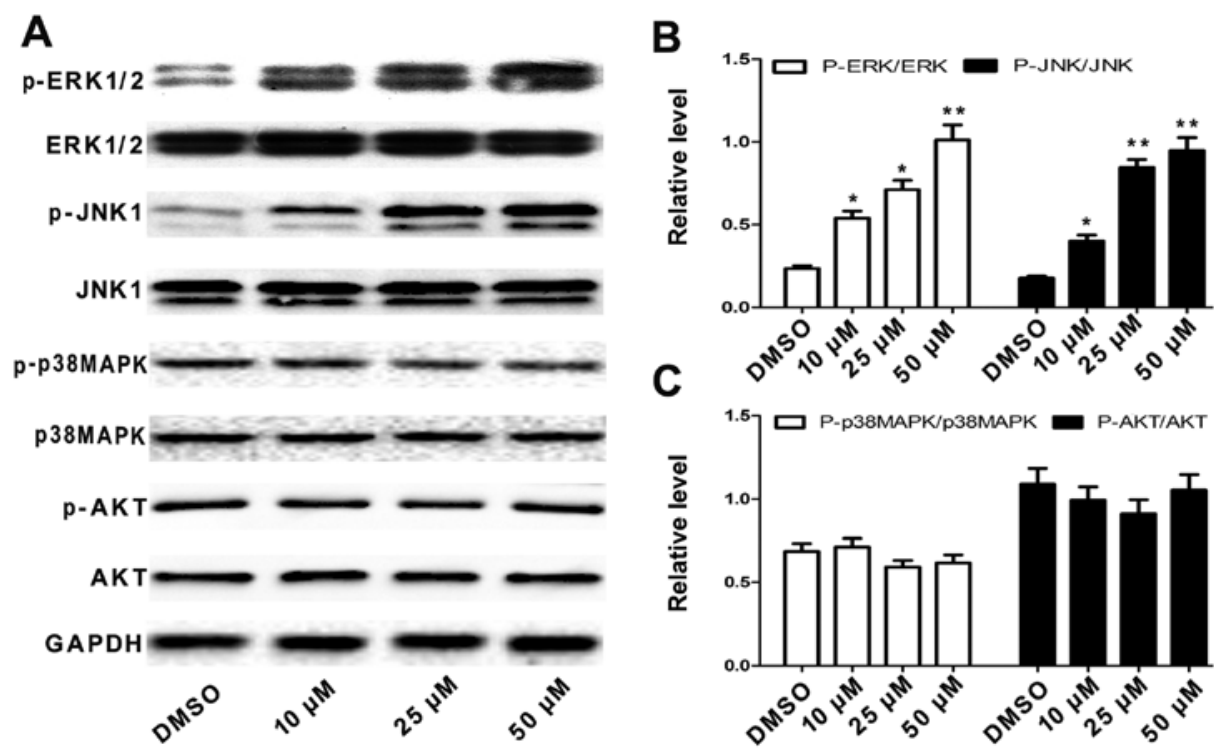

Figure 5. Activation of ERK1/2 and JNK1/2 was increased in angelicin-treated A549 cells. (A) A549 cells were treated with different concentration of angelicin and western blotting were applied for analysis of phosphorylation of ERK, JNK, p38 and AKT. (B and C) Results were quantified by densitometry analysis of the bands from (A) and then normalization to GAPDH protein. ${ }^{*} \mathrm{P}<0.05,{ }^{* *} \mathrm{P}<0.01$ compared with DMSO, $\mathrm{n}=3$. JNK, c-Jun $\mathrm{NH}_{2}$-terminal protein kinase; ERK, extracellular signal-regulated kinase.

treatments significantly attenuated angelicin-induced JNK and ERK activation (Fig. 6A and B). The results suggested that JNK and ERK activation may play a crucial role in angelicin-mediated effects on A549 cells. Next, the cell cycle, apoptosis and migration of A549 cells were examined with or without SP600125 and U0126 pretreatments. As shown 

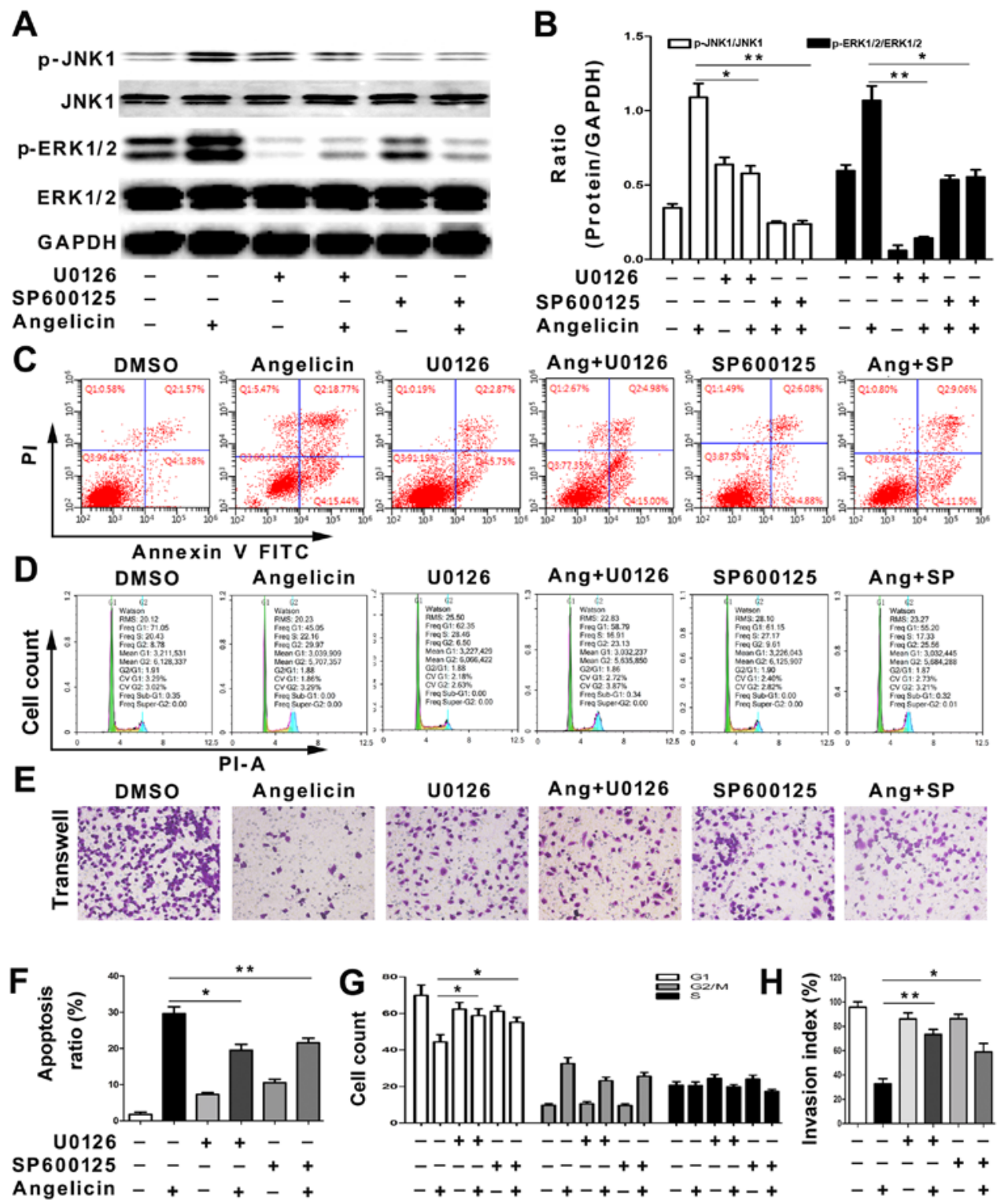

Figure 6. Angelicin inhibits A549 cell growth and migration through regulating JNK and ERK pathways. A549 cells were pretreated with $25 \mu \mathrm{M}$ SP600125 or U0126 for $45 \mathrm{~min}$ and then treated with $50 \mu \mathrm{M}$ angelicin for $24 \mathrm{~h}$, respectively. (A) The expression levels of p-ERK1/2/ERK1/2 and p-JNK1/2/JNK1/2 were measured by western blotting. (B) Results were quantified by densitometry analysis of the bands from (A) and then normalization to GAPDH protein. (C) Representative flow cytometry scatter plots of PI (y-axis) vs. Annexin V-FITC (x-axis). (D) Representative flow cytometry cell cycle of PI. (E) The invasive ability was evaluated by a Transwell invasion assay (x200 magnification). (F) Quantitative results of (C). (G) Quantitative results of (D). (H) Quantitative results of (E). ${ }^{*} \mathrm{P}<0.05,{ }^{* *} \mathrm{P}<0.01$ compared with control, $\mathrm{n}=4$. JNK, c-Jun $\mathrm{NH}_{2}$-terminal protein kinase; ERK, extracellular signal-regulated kinase; PI, propidium iodide; FITC, fluorescein isothiocyanate.

in Fig. 6C-H, the SP600125 and U0126 treatments partially alleviated the angelicin-induced apoptosis, cell cycle arrest and migration inhibition of A549 cells. The results indicated that angelicin inhibits A549 cell growth and migration through both the ERK and JNK pathways.

Angelicin inhibits A549 cell growth and metastasis in vivo. We further evaluated the antitumor effect of angelicin in A549 cancer cells by utilizing nude mouse xenograft models. As anticipated, tumor size and weight decreased significantly in the angelicin group compared with the control group (Fig. 7A). The anti-metastatic effects of angelicin were further assessed with an in vivo model of lung metastasis. As shown in Fig. 7B, angelicin had an inhibitory effect on A549 tumor metastasis to the lungs compared with the control group. TUNEL labeling and the expression levels of MMP2, MMP9 and E-cadherin were evaluated immunohistochemically (Fig. 7C). Angelicin significantly increased the ratio of TUNEL-positive cells, which is indicative of apoptosis, in metastatic nodules of the lung metastasis model. Furthermore, the angelicin treatment resulted in decreased expression levels of MMP2 and MMP9 and increased expression of E-cadherin, which was consistent with the in vitro study. These results indicate that angelicin can significantly inhibit A549 cell growth and metastasis in vivo.

\section{Discussion}

Recently, angelicin has gained much attention due to its biological multifunctionality. In the present study, angelicin suppressed the growth and metastasis of A549 human lung 

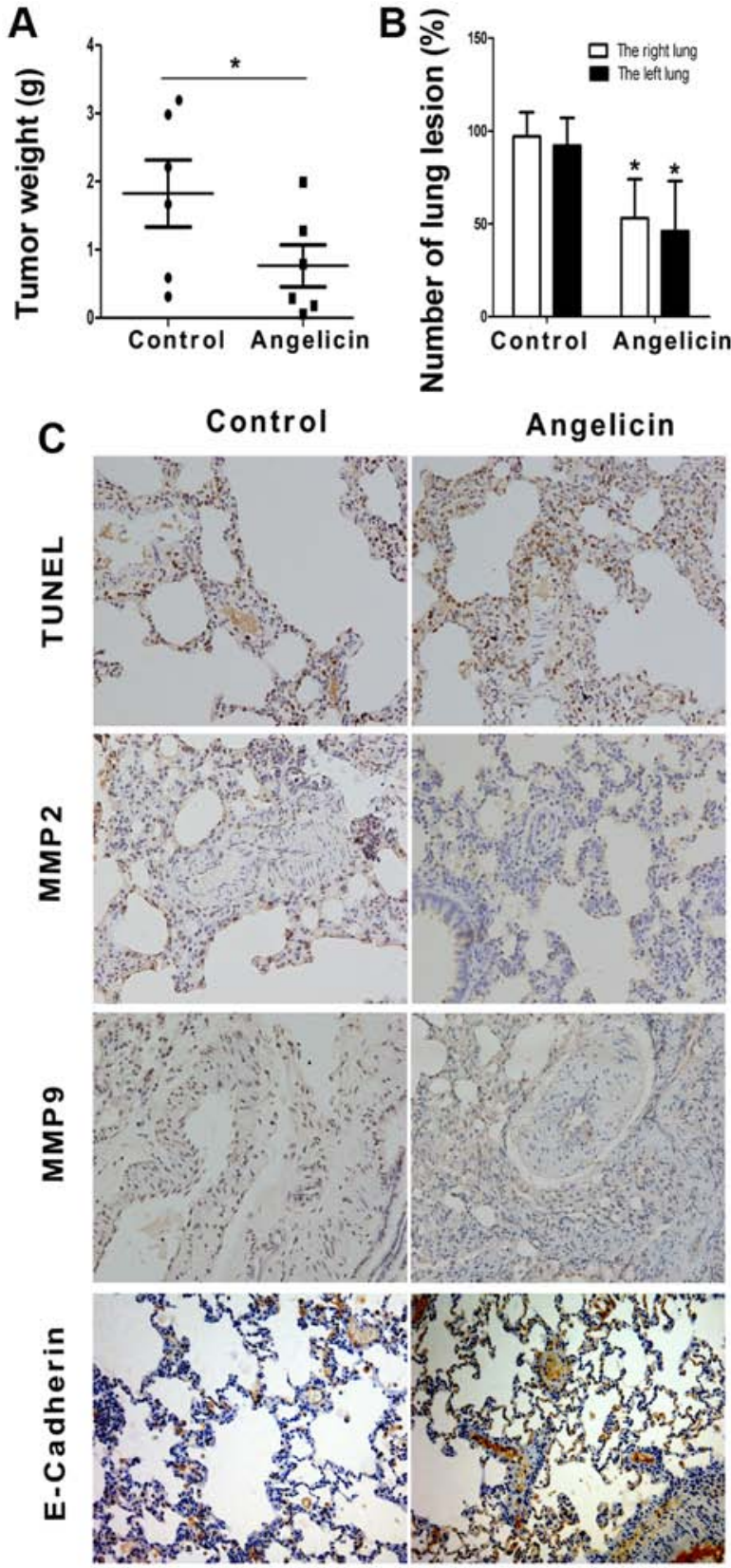

Figure 7. Angelicin suppresses cancer growth and lung metastasis of A549 cells in vivo. (A) Effect of angelicin $(100 \mathrm{mg} / \mathrm{kg}$ ) on the tumor growth was investigated in the model of A549 subcutaneous transplantation. (B) Effect of angelicin $(100 \mathrm{mg} / \mathrm{kg})$ on the number of lung metastatic nodules. (C) The expression of TUNEI, E-cadherin, MMP2 and MMP9 proteins were analyzed by immunohistochemical assay (x400 magnification). Each experiment was performed thrice. ${ }^{*} \mathrm{P}<0.05$ compared with control, $\mathrm{n}=6$. MMP, matrix metalloproteinase.

adenocarcinoma cells both in vitro and in vivo. The mechanisms underlying this process involve apoptosis induction, cell cycle arrest at the $\mathrm{G} 2 / \mathrm{M}$ phase and migration inhibition. Additionally, angelicin was able to regulate the expression of apoptosis-, cell cycle-, and migration-related proteins and activate caspase activity. Moreover, the effects exerted by angelicin in A549 cells may be regulated by ERK and JNK pathway modulation. Angelicin was capable of inducing apoptosis, inhibiting growth and hindering metastasis in vivo.

In this study, we observed that angelicin strongly inhibited A549 cell growth. As shown in MTT, the $\mathrm{IC}_{50}$ values of angelicin against A549 cells was nearly $50 \mu \mathrm{mol}$. Further, we found $10 \mu \mathrm{mol}$ angelicin exhibited inhibitory effects on cell proliferation $(\mathrm{P}<0.05)$. Thus, angelicin concentrations of 10 , 25 and $50 \mu \mathrm{mol}$ were selected for the subsequent assays.

The anticancer effects of therapeutics have been observed to be linked to the process of inducing apoptosis (19). Apoptosis is a process leading to programmed cell death that is highly regulated by several signaling pathways, including those of caspases and the Bcl-2 family (20). Bax and Bcl-2 are two key proteins of the Bcl-2 family and are critical for cells to undergo apoptosis (21). In this study, we observed significant changes in Bax and Bcl-2 protein expression after angelicin treatment, which occurred in a dose-dependent manner. Specifically, the ratio of Bax to Bcl-2 markedly increased, which is considered the driving force of apoptosis (22). The caspase family consists of cysteine-containing proteolytic enzymes that have a potent role in executing apoptosis (23). In the present study, angelicin was shown to induce caspase- 3 and caspase- 9 activation in a dose-dependent manner. Thus, we conclude that angelicin may induce apoptosis by regulating these apoptosis-related proteins.

Cell cycle disorder is known to contribute to cancer cell growth and the development of various types of cancer (24). Thus, arresting the cell cycle is considered a very effective method for eradicating tumor cells. The G2/M phase is an important checkpoint in the cell cycle that prevents the initiation of mitosis until DNA damaged during replication is repaired (25). The majority of the cells treated with angelicin were arrested in the $\mathrm{G} 2 / \mathrm{M}$ phase. Additionally, cell cycle progression is tightly related to various cyclins and cyclin-dependent kinases (CDKs), such as cyclin B1, cyclin E1 and Cdk2 (26). We examined the modulation of these key proteins involved in cell cycle arrest and observed that cyclin B1, cyclin E1 and Cdk2, master regulators of the cell cycle, were efficiently modulated by angelicin. These data indicate that angelicin could inhibit cell growth by regulating cyclin $\mathrm{B} 1$, cyclin $\mathrm{E} 1$ and $\mathrm{Cdk} 2$ expression levels, thereby arresting the cell cycle in the $\mathrm{G} 2 / \mathrm{M}$ phase.

Tumor metastasis is a major cause of death in NSCLC patients; thus, it is crucial to identify promising anti-metastatic agents to prevent or inhibit metastasis (27). In this study, we found that angelicin significantly inhibited A549 cell invasion and migration. Additionally, the expression levels of several crucial metastasis genes were assessed, including E-cadherin, MMP2 and MMP9, which play key roles in regulating cancer cell invasion and metastasis (28). Abnormal expression levels of E-cadherin, MMP2 and MMP9 in tumor cells will lead to decreased adhesion as well as enhanced migration and invasion, thus promoting cancer progression (29). Our results showed that angelicin increased the expression of E-cadherin and strongly decreased the expression of MMP2 and MMP9, which suggested that angelicin would be an effective agent against NSCLC metastasis.

Previous studies have demonstrated that the MAPK and AKT signaling pathways play important roles in regulating apoptosis, the cell cycle and migration (30). The present study evaluated the effects of angelicin on the phosphorylation of signaling molecules in these pathways in A549 cells. The MAPK family includes JNC/stress-activated protein kinase (SAPK), p38 MAPK and ERK (31). In this study, we showed that angelicin increased JNK and ERK phosphorylation in A549 cells in a dose-dependent manner, while the total 
protein level remained steady; however, angelicin had no effect on p38 MAPK and AKT activation. Furthermore, treatment with a JNK1/2-specific inhibitor (SP600125) or an ERK1/2 inhibitor (U0126) effectively alleviated the angelicin-induced apoptosis, cell cycle arrest and migration inhibition in A549 cells. These findings suggest that JNK and ERK activation plays a critical role in angelicin-induced apoptosis, cell cycle arrest and migration inhibition in NSCLC A549 cells.

In addition to investigating the in vitro anticancer and anti-metastatic activity of angelicin, we assessed the antitumor and anti-metastatic effects of angelicin in vivo. The angelicin treatment decreased the volume and weight of subcutaneous tumor mass in this model. Moreover, in the lung metastasis model, we found that treatment with angelicin not only inhibited the formation of metastatic nodules but also induced apoptosis and decreased the expression of migration-related proteins in the lungs.

In conclusion, angelicin exerts antitumor and anti-metastatic activity by inducing apoptosis, arresting the cell cycle and inhibiting migration in human lung carcinoma A549 cells. The mechanisms underlying these effects are associated with activation of the JNK and ERK pathways. Therefore, angelicin may be a potential therapeutic agent for the treatment of human lung cancer.

\section{References}

1. Pusceddu S, Lo Russo G, Macerelli M, Proto C, Vitali M, Signorelli D, Ganzinelli M, Scanagatta P, Duranti L, Trama A, et al: Diagnosis and management of typical and atypical lung carcinoids. Crit Rev Oncol Hematol 100: 167-176, 2016.

2. Villaruz LC and Socinski MA: Is there a role of nab-paclitaxel in the treatment of advanced non-small cell lung cancer? The data suggest yes. Eur J Cancer 56: 162-171, 2016.

3. Bremnes RM, Busund LT, Kilvær TL, Andersen S, Richardsen E, Paulsen EE, Hald S, Khanehkenari MR, Cooper WA, Kao SC, et al: The role of tumor-infiltrating lymphocytes in development, progression, and prognosis of non-small cell lung cancer. J Thorac Oncol 11: 789-800, 2016.

4. Sibille A, Paulus A, Martin M, Bourhaba M, Barthélemy N, Radermecker M, Corhay JL, Louis R and Duysinx B: [Management of non-small cell lung cancer]. Rev Med Liege 70: 432-441, 2015 (In French).

5. Shea M, Costa DB and Rangachari D: Management of advanced non-small cell lung cancers with known mutations or rearrangements: latest evidence and treatment approaches. Ther Adv Respir Dis 10: 113-129, 2016.

6. Sidaway P: CNS cancer: distinct subtypes of ATRTs observed. Nat Rev Clin Oncol 13: 264, 2016.

7. Lee WS, Park YL, Kim N, Oh HH, Son DJ, Kim MY, Oak CY, Chung CY, Park HC, Kim JS, et al: Myeloid cell leukemia-1 is associated with tumor progression by inhibiting apoptosis and enhancing angiogenesis in colorectal cancer. Am J Cancer Res 5 : 101-113, 2014.

8. Li HX, Fu XJ, Yang K, Chen D, Tang H and Zhao Q: The clock gene PER1 suppresses expression of tumor-related genes in human oral squamous cell carcinoma. Oncotarget 7: 20574-20583, 2016

9. Jara P, Calyeca J, Romero Y, Plácido L, Yu G, Kaminski N, Maldonado V, Cisneros J, Selman M and Pardo A: Matrix metalloproteinase (MMP)-19-deficient fibroblasts display a profibrotic phenotype. Am J Physiol Lung Cell Mol Physiol 308 L511-L522, 2015.

10. Pan B, Ren H, Ma Y, Liu D, Yu B, Ji L, Pan L, Li J, Yang L, Lv X, et al: High-density lipoprotein of patients with type 2 diabetes mellitus elevates the capability of promoting migration and invasion of breast cancer cells. Int J Cancer 131: 70-82, 2012

11. Rahman MA, Kim NH, Yang H and Huh SO: Angelicin induces apoptosis through intrinsic caspase-dependent pathway in human SH-SY5Y neuroblastoma cells. Mol Cell Biochem 369: 95-104, 2012.

12. Bordin F, Dall'Acqua F and Guiotto A: Angelicins, angular analogs of psoralens: chemistry, photochemical, photobiological and phototherapeutic properties. Pharmacol Ther 52: 331-363, 1991.
13. Mosti L, Lo Presti E, Menozzi G, Marzano C, Baccichetti F, Falcone G, Filippelli W and Piucci B: Synthesis of angelicin heteroanalogues: preliminary photobiological and pharmacological studies. Farmaco 53: 602-10, 1998.

14. Lampronti I, Bianchi N, Borgatti M, Fibach E, Prus E and Gambari R: Accumulation of gamma-globin mRNA in human erythroid cells treated with angelicin. Eur J Haematol 71: 189-195, 2003.

15. Jeong D, Watari K, Shirouzu T, Ono M, Koizumi K, Saiki I, Kim YC, Tanaka C, Higuchi R and Miyamoto T: Studies on lymphangiogenesis inhibitors from Korean and Japanese crude drugs. Biol Pharm Bull 36: 152-157, 2013.

16. Ryu BJ, Lee H, Kim SH, Heo JN, Choi SW, Yeon JT, Lee J, Lee J, Cho JY, Kim SH, et al: PF-3758309, p21-activated kinase 4 inhibitor, suppresses migration and invasion of A549 human lung cancer cells via regulation of $\mathrm{CREB}, \mathrm{NF}-\kappa \mathrm{B}$, and $\beta$-catenin signalings. Mol Cell Biochem 389: 69-77, 2014.

17. Lee AY, Fan CC, Chen YA, Cheng CW, Sung YJ, Hsu CP and Kao TY: Curcumin inhibits invasiveness and epithelial-mesenchymal transition in oral squamous cell carcinoma through reducing matrix metalloproteinase 2,9 and modulating p53-E-cadherin pathway. Integr Cancer Ther 14: 484-490, 2015.

18. Xiang T, Fei R, Wang Z, Shen Z, Qian J and Chen W: Nicotine enhances invasion and metastasis of human colorectal cancer cells through the nicotinic acetylcholine receptor downstream p38 MAPK signaling pathway. Oncol Rep 35: 205-210, 2016.

19. Lee D, Kim IY, Saha S and Choi KS: Paraptosis in the anti-cancer arsenal of natural products. Pharmacol Ther 162: 120-133, 2016.

20. Ghate NB, Hazra B, Sarkar R, Chaudhuri D and Mandal N: Alteration of $\mathrm{Bax} / \mathrm{Bcl}-2$ ratio contributes to Terminalia belerica-induced apoptosis in human lung and breast carcinoma. In Vitro Cell Dev Biol Anim 50: 527-537, 2014.

21. Saiprasad G, Chitra P, Manikandan R and Sudhandiran G: Hesperidin induces apoptosis and triggers autophagic markers through inhibition of Aurora- $A$ mediated phosphoinositide-3-kinase/Akt/mammalian target of rapamycin and glycogen synthase kinase- 3 beta signalling cascades in experimental colon carcinogenesis. Eur J Cancer 50: 2489-2507, 2014.

22. Liu F, Jiang YJ, Zhao HJ, Yao LQ and Chen LD: Electroacupuncture ameliorates cognitive impairment and regulates the expression of apoptosis-related genes $\mathrm{Bcl}-2$ and $\mathrm{Bax}$ in rats with cerebral ischaemia-reperfusion injury. Acupunct Med 33: 478-484, 2015.

23. Ashkenazi A and Salvesen G: Regulated cell death: signaling and mechanisms. Annu Rev Cell Dev Biol 30: 337-356, 2014

24. Carneiro BA, Meeks JJ, Kuzel TM, Scaranti M, Abdulkadir SA and Giles FJ: Emerging therapeutic targets in bladder cancer. Cancer Treat Rev 41: 170-178, 2015.

25. Asanagi M, Yamada S, Hirata N, Itagaki H, Kotake Y, Sekino Y and Kanda Y: Tributyltin induces $\mathrm{G} 2 / \mathrm{M}$ cell cycle arrest via $\mathrm{NAD}(+)$-dependent isocitrate dehydrogenase in human embryonic carcinoma cells. J Toxicol Sci 41: 207-215, 2016.

26. Arooz T, Yam CH, Siu WY, Lau A, Li KK and Poon RY: On the concentrations of cyclins and cyclin-dependent kinases in extracts of cultured human cells. Biochemistry 39: 9494-9501, 2000.

27. Zhen Q, Liu J, Gao L, Liu J, Wang R, Chu W, Zhang Y, Tan G, Zhao X and Lv B: MicroRNA-200a targets EGFR and c-Met to inhibit migration, invasion, and gefitinib resistance in non-small cell lung cancer. Cytogenet Genome Res 146: 1-8, 2015.

28. Lemieux E, Bergeron S, Durand V, Asselin C, Saucier C and Rivard N: Constitutively active MEK1 is sufficient to induce epithelial-to-mesenchymal transition in intestinal epithelial cells and to promote tumor invasion and metastasis. Int J Cancer 125: 1575-1586, 2009.

29. Qin L, Liao L, Redmond A, Young L, Yuan Y, Chen H, O'Malley BW and Xu J: The AIB1 oncogene promotes breast cancer metastasis by activation of PEA3-mediated matrix metalloproteinase 2 (MMP2) and MMP9 expression. Mol Cell Biol 28: 5937-5950, 2008.

30. Radnai B, Antus C, Racz B, Engelmann P, Priber JK, Tucsek Z, Veres B, Turi Z, Lorand T, Sumegi B, et al: Protective effect of the poly(ADP-ribose) polymerase inhibitor PJ34 on mitochondrial depolarization-mediated cell death in hepatocellular carcinoma cells involves attenuation of c-Jun $\mathrm{N}$-terminal kinase-2 and protein kinase B/Akt activation. Mol Cancer 11: $34,2012$.

31. Haas N, Riedt T, Labbaf Z, Baßler K, Gergis D, Fröhlich H, Gütgemann I, Janzen V and Schorle H: Kit transduced signals counteract erythroid maturation by MAPK-dependent modulation of erythropoietin signaling and apoptosis induction in mouse fetal liver. Cell Death Differ 22: 790-800, 2015. 